\title{
Equilibrium sedimentation profiles of charged colloidal suspensions
}

\author{
Gabriel Téllez and Thierry Biben \\ Laboratoire de Physique (Unité Mixte de Recherche du Centre National de la Recherche \\ Scientifique - UMR 5672) Ecole Normale Supérieure de Lyon, 69364 Lyon cedex 07, \\ France
}

\begin{abstract}
We investigate the sedimentation equilibrium of a charge stabilized colloidal suspension in the regime of low ionic strength. We analyze the asymptotic behaviour of the density profiles on the basis of a simple Poisson-Boltzmann theory and show that the effective mass we can deduce from the barometric law corresponds to the actual mass of the colloidal particles, contrary to previous studies.
\end{abstract}

\section{INTRODUCTION}

Under the action of gravity a colloidal suspension sediments to form a stratified fluid. The equilibrium density profile of the colloidal particles results from the balance between the gravitational force and thermodynamic forces as derived from the free energy of the system. The density profiles usually exhibits a dense layer of colloidal particles at the bottom of the container above which a light cloud of colloidal particles floats. In this last regime, the density of particles is small enough to treat the fluid as an ideal gas. Under the reasonable assumption that density gradients can be neglected, the equilibrium colloidal density obey the well known barometric law:

$$
\rho_{\mathrm{col}}(z)=\rho_{\mathrm{col}}^{0} \exp \left(-z / l_{g}\right)
$$

Here, $\rho_{\text {col }}(z)$ denotes the density profile of the colloidal particles, $z$ is the altitude and $l_{g}=(\beta M g)^{-1}$ is the gravitational length where $\beta=\left(k_{\mathrm{B}} T\right)^{-1}$ is the inverse temperature, $M$ is the buoyant mass of a colloidal particle and $g$ the intensity of the gravitational field. This exponential law is of practical interest since it gives a prescription for the measurement of the buoyant mass $M$ of the particles. However a recent experimental study of the sedimentation profiles of strongly de-ionized charged colloidal suspensions [1] lead the authors to challenge the validity of this barometric law. An exponential behaviour was indeed observed in the asymptotic regime, but the measured gravitational length $l_{g}^{*}$ could differ significantly from the expected one (a factor of two). $l_{g}^{*}$ was found to systematically overestimate the actual value $l_{g}$, with the result that the buoyant mass measured within these experiments is systematically reduced compared to the known buoyant mass of the particles.

Some theoretical efforts have been made to study this problem. First Biben and Hansen [2] solved numerically the problem in a mean field approach, but unfortunately due to numerical difficulties the samples height considered where of the order of the micron while in the experiment the samples height are of the order of

LPENS-Th 10/99 
the centimeter. As a consequence, the dilute region at high altitude could not be studied in this approach. Nevertheless the numerical results show a positive charge density at the bottom of the container and a negative charge at the top while the bulk of the container is neutral. This result show that a non-zero electric field exists in the bulk of the container and acts against gravity for the colloids.

More recently one of the authors studied a two-dimensional solvable model for this problem [3]. This model is not very realistic (the valency of the colloids was $Z=1$ and there was no added salt) but has the nice feature of being exactly solvable analytically. It confirmed the condenser effect noticed for small height containers in Ref. [2]. For large height containers it showed a new interesting phenomenon: while there is still a positive charge density at the bottom of the container, the negative charge density is not any more at the top of the container floating but at some altitude. Interestingly, the analytical expression for the density profiles in the asymptotic regime predicts a decay in $\exp \left(-z / l_{g}\right) / z$ for the colloidal density. Besides the $1 / z$ factor that cannot be explained by a mean field approach, no mass reduction is predicted by this model. However one should be cautious when comparing two-dimensional systems to the three dimensional case because the density in not relevant in two-dimensional Coulomb systems: no matter how small the density is the system is always coupled, the ideal gas regime is never attained. For this reason a decay of the density similar to the one of an ideal gas is in itself surprising in two dimensions.

Lately new results based on an approximate version of the model introduced in reference [2] lead the authors of these studies [4.5] to conclude that the mean-field approach was indeed able to predict a mass reduction in the asymptotic regime. Here we present some new results about this problem treated under the PoissonBoltzmann approximation, and show that it is indeed not the case.

\section{THE MODEL AND THE POISSON-BOLTZMANN APPROXIMATION}

Let us consider some colloidal particles (for example some latex spheres) in a solution with some amount of added salt. In a polar solvent like water the colloids release some counterions and therefore acquire a surface electric charge $Z e$ ( $Z$ is a entire number usually positive and $-e$ is the charge of the electron). We consider that the colloidal sample is monodisperse, all colloids have the same valency $Z$, and that the counterions and the salt cations are both monovalent and therefore we shall not make any distinction between cations coming from the colloids and salt cations. We then consider a three-component system composed of colloidal particles with electric charge $Z e$ and mass $M$, counterions with charge $-e$ and coions with charge $+e$. We shall neglect the masses of the counterions and coions when compared with the mass of the colloids. The solvent shall be considered in a primitive model representation as a continuous medium of relative dielectric permittivity $\epsilon$ (for water at room temperature $\epsilon \approx 80$ ). The system is in a container of height $h$, the bottom of the container is at $z=0$ altitude. We consider that the system is invariant in the horizontal directions. The density profiles of each species are denoted by $\rho_{\text {col }}(z), \rho_{+}(z)$ and $\rho_{-}(z)$ ( $z$ is the vertical coordinate) for the colloids, the cations and the anions respectively at equilibrium. Let us define the electric charge density (in units of $e$ ) $\rho=Z \rho_{\text {col }}-\rho_{-}+\rho_{+}$and the electric potential $\Phi$, solution of the Poisson equation

$$
\frac{d^{2} \Phi}{d z^{2}}(z)=-\frac{4 \pi}{\epsilon} e \rho(z)
$$

It is instructive to recall that the Poisson-Boltzmann equation can be derived from the minimization of the free energy density functional 


$$
\begin{aligned}
\mathcal{F}\left[\rho_{\mathrm{col}}, \rho_{+}, \rho_{-}\right]= & \sum_{i \in\{\mathrm{col},+,-\}} \int_{0}^{h} k_{\mathrm{B}} T \rho_{i}(z)\left[\ln \left(\lambda_{i}^{3} \rho_{i}(z)\right)-1\right] d z \\
& +\int_{0}^{h} M g z \rho_{\mathrm{col}}(z) d z+\frac{1}{2} \int_{0}^{h} e \rho(z) \Phi(z)
\end{aligned}
$$

where $\lambda_{i}$ is the de Broglie wavelength of species $i$. Minimization of the grand potential with respect to the densities: $\delta \mathcal{F} / \delta \rho_{i}(z)-\mu_{i}=0$, where $\mu_{i}$ is the chemical potential of species $i$, yields

$$
\begin{aligned}
\rho_{\mathrm{col}}(z) & =\rho_{\mathrm{col}}^{0} \exp (-\beta Z e \Phi(z)-\beta M g z) \\
\rho_{+}(z) & =\rho_{+}^{0} \exp (-\beta e \Phi(z)) \\
\rho_{-}(z) & =\rho_{-}^{0} \exp (\beta e \Phi(z))
\end{aligned}
$$

We shall work in the canonical ensemble, the prefactors $\rho_{i}^{0}$ which depend on the chemical potentials $\mu_{i}$ are determined by the normalizing conditions

$$
\int_{0}^{h} \rho_{i}(z) d z=N_{i}
$$

where $N_{i}$ is the total number of particles per unit area of species $i$. The system is globally neutral so we have $Z N_{\mathrm{col}}-N_{-}+N_{+}=0$.

Let us introduce the following notations: $l_{g}=(\beta M g)^{-1}$ is the gravitational length of the particles, $l=\beta e^{2} / \epsilon$ is the Bjerrum length, $\phi=\beta e \Phi$ is the dimensionless electric potential and $\kappa_{i}=\left(4 \pi l N_{i} / h\right)^{1 / 2} \cdot \kappa_{ \pm}^{-1}$ are the Debye lengths associated to the counterions and the coions and $\left(Z \kappa_{\mathrm{col}}\right)^{-1}$ is the Debye length associated to the colloidal particles. For a quantity $q(z)$ depending on the altitude, let us define its mean value $\langle q\rangle=\int_{0}^{h} q(z) d z / h$. With these notations equations (2.1) and (2.3) yield the modified Poisson-Boltzmann equation

$$
\frac{d^{2} \phi}{d z^{2}}(z)=-Z \kappa_{\mathrm{col}}^{2} \frac{e^{-Z \phi(z)-z / l_{g}}}{\left\langle e^{-Z \phi\left(z^{\prime}\right)-z^{\prime} / l_{g}}\right\rangle}+\kappa_{-}^{2} \frac{e^{\phi(z)}}{\left\langle e^{\phi\left(z^{\prime}\right)}\right\rangle}-\kappa_{+}^{2} \frac{e^{-\phi(z)}}{\left\langle e^{-\phi\left(z^{\prime}\right)}\right\rangle}
$$

From Eq. (2.5) it is clear that the problem has the following scale invariance: if $\phi(z)$ is a solution of $(2.5)$ then $\phi(\alpha z)$ is a solution of the problem with the rescaled lengths $\alpha l_{g}$ and $\alpha \kappa_{i}^{-1}$.

The advantage of the density functional formulation of the problem is that it allows for systematic corrections to the Poisson-Boltzmann approximation. For instance, one may be interested in the effect of the finite size of the macroions. Let $\sigma$ be the diameter of the colloids, $\eta_{\text {col }}=\pi \sigma^{3} \rho_{\text {col }} / 6$ the volume fraction of colloids and $\rho_{ \pm}^{*}=\rho_{ \pm} /\left(1-\eta_{\mathrm{col}}\right)$ the effective densities of the microions. Then, the finite size of the colloids can be accounted in a local density approximation (LDA) by adding to the free energy density functional (2.2) the free energy excess term

$$
\int_{0}^{h} f_{\text {exec }}\left(\rho_{\operatorname{col}}(z)\right) d z
$$

where $f_{\text {exec }}\left(\rho_{\text {col }}\right)$ is the excess free energy of a hard sphere fluid derived by the Carnahan-Starling equation of state [6]

$$
f_{\text {exec }}\left(\rho_{\mathrm{col}}\right)=k_{\mathrm{B}} T \rho_{\mathrm{col}} \frac{4 \eta_{\mathrm{col}}-3 \eta_{\mathrm{col}}^{2}}{\left(1-\eta_{\mathrm{col}}\right)^{2}}
$$

and by replacing in (2.2) the entropy term of the microions by 


$$
k_{\mathrm{B}} T \int_{0}^{h} \rho_{ \pm}(z)\left(\ln \left(\lambda_{ \pm}^{3} \rho_{ \pm}^{*}(z)\right)-1\right) d z .
$$

Minimization of this new free energy functional gives the modified version of Eqs. (2.3)

$$
\begin{aligned}
\rho_{\mathrm{col}}(z)= & \rho_{\mathrm{col}}^{0} \exp (-\beta Z e \Phi(z)-\beta M g z) \\
& \times \exp \left[\frac{3 \eta_{\mathrm{col}}(z)^{3}-9 \eta_{\mathrm{col}}(z)^{2}+8 \eta_{\mathrm{col}}(z)}{\left(1-\eta_{\mathrm{col}}(z)\right)^{3}}+\frac{\pi \sigma^{3}}{6}\left(\rho_{+}^{*}(z)+\rho_{-}^{*}(z)\right)\right] \\
\rho_{+}(z)= & \rho_{+}^{0}\left(1-\eta_{\mathrm{col}}(z)\right) \exp (-\beta e \Phi(z)) \\
\rho_{-}(z)= & \rho_{-}^{0}\left(1-\eta_{\mathrm{col}}(z)\right) \exp (\beta e \Phi(z))
\end{aligned}
$$

There are several length scales in this problem: the gravitational length of the colloids $l_{g}$, the Debye or screening length, the height $h$ of the container and eventually the hard core diameter of the particules. In a realistic case $h$ is of the order of the centimeter, $l_{g}$ of the order of $0.1 \mathrm{~mm}$, the screening length of the order of $10 \mathrm{~nm}$. We are faced to a practical numerical problem, when we will transpose the problem to a lattice, the lattice spacing should be smaller than all the physical lengths, but since $h$ is much larger than the others lengths, the number of sites in the lattice should be very high (of order $10^{6}$ ). A possible approach to deal with this problem is to study small containers as in Ref. [2]. In this paper we want to study the case of high containers so we will consider very deionized systems in which the screening length is of the order of $0.1 \mathrm{~mm}$, much larger than in usual physical cases, the other lengths taking "physical" values. That way the necessary number of points in the lattice will be reasonable (a few hundreds). Also, since the screening length is so large, the hard core of the macroions will not change the results drastically from the case of point particles so we will concentrate from now on on the Poisson-Boltzmann problem for point particles (Eq. 2.5).

\section{RESULTS}

Equation (2.5) is solved numerically by an iterative method [7]. Using the Green function of the one-dimensional Laplacian

$$
G\left(z, z^{\prime}\right)=\frac{1}{2}\left|z-z^{\prime}\right|
$$

the Poisson equation (2.1) can be written as

$$
\phi(z)=-4 \pi l \int_{0}^{h} G\left(z, z^{\prime}\right) \rho\left(z^{\prime}\right) d z^{\prime}
$$

Starting with an arbitrary electric potential, one can compute the corresponding density profiles using Eqs. (2.3) and derive a new electric potential using Eq. (3.2),

then reiterate the process until a stationary solution is attained. In practice instead of using the new potential directly for the next iteration a mixing of the old and new densities is used.

\section{A. Generic results}

As stated before we have to consider very deionized systems in which the Bjerrum length $l$ is smaller than $10^{-6} \AA$ (the physical value of $l$ for water at room temperature 
is $7 \AA$ ). Figure 1 shows the density profiles of each species, the charge density, the electric potential and the electric field profile of a typical sample with the following parameters: $l=7 \cdot 10^{-8} \AA, l_{g}=0.128 \mathrm{~mm}, h=30 \mathrm{~mm}, Z=100$, a salt concentration $C_{\text {salt }}=0.1 \mathrm{mMol} / \mathrm{l}$ and a mean colloidal volume fraction $\bar{\eta}_{\mathrm{col}}=0.12$ (we consider that the particles have a hard core diameter $\sigma=180 \mathrm{~nm}$ to express the colloidal density as a volume fraction in order to use units familiar with the experiments but we do not account for hard core effects in the Poisson-Boltzmann equation).

The log plot of colloidal density profiles is similar to the experimental ones [1]. In the bottom there is a slow decay whereas at high altitudes there is a faster barometric decay. Since we did not take into account the hard core of the particles in the theory we do not find the discontinuity in the density profiles near the bottom of the sample observed in the experiments [1], due to the phase transition of the colloids from an amorph solid to a fluid. At very low altitudes (near the bottom, very high volume fractions) the Poisson-Boltzmann theory is not valid.

The charge density profile confirms the results of Ref. [3], that there is a strong accumulation of positive charges at the bottom of the container while there is a cloud of negative charge density floating at some altitude $z^{*}$. There are clearly two neutral regions in the container: one at low altitude between the positive charge density at the bottom and the negative cloud, in which a non-vanishing electric field exists and a second neutral region at high altitude, over the negative cloud. The electric field in the lower region acts against gravity for the colloids therefore, as seen in the log plot of the colloids density profile, the decay is much slower than the one for an ideal neutral gas. Numeric results for other series of samples suggest that this electric field is proportional to $M g / Z$. In the upper region the colloidal density drops exponentially as $\exp \left(-z / l_{g}\right)$ since the electric potential is almost constant and the electric field vanishes.

Since the different densities vary with the altitude we can define a local screening length which depends on the altitude by

$$
\lambda(z)=\left(4 \pi l\left(Z^{2} \rho_{\mathrm{col}}(z)+\rho_{+}(z)+\rho_{-}(z)\right)\right)^{-1 / 2}
$$

The two regions of the sediment are caracterized by a very different behavior of this local screening length. In the lower region the colloidal density is so high that $Z^{2} \rho_{\text {col }}(z) \gg \rho_{+}(z)+\rho_{-}(z)$. In that region the colloids dominate the screening length. On the other hand in the upper region the colloidal density is very small and salt now controles the screening length which is then constant since at high altitudes the cations and anions densities are almost constant and equal to the mean salt concentration as seen in figure If. It is interesting to notice that electric charges accumulate in the intermediate region around $z^{*}$ where there is a change of regime, in agreement with macroscopic electrostatics principles.

The preceding remark allows us to understand how the physical parameters (mean volume fraction of colloids, mass of the colloids, amount of added salt) will modify the altitude $z^{*}$ which separates the two regions. For example if we add more salt, $z^{*}$ will diminish since we reach sooner the regime where $Z^{2} \rho_{\text {col }}(z)<\rho_{+}(z)+\rho_{-}(z)$. We have computed the density profiles in several other cases changing the values of the parameters in order to find the depency of $z^{*}$ in these parameters. Our numerical results suggest that

$$
z^{*}=-\frac{c_{1}}{\sqrt{l C_{\mathrm{salt}}}}+a_{2} \frac{Z \sqrt{N_{\mathrm{col}} l_{g}}}{\sqrt{C_{\mathrm{salt}}}}
$$

with $c_{1}=0.15 \pm 0.05$ and $a_{2}=1.0 \pm 0.1$. The preceding equation can be written in a more attracting way, introducing the screening length associated to the salt $\lambda_{\text {salt }}=\left(4 \pi l C_{\text {salt }}\right)^{-1 / 2}$ and the effective screening length associated to the colloids $\lambda_{\mathrm{col}}^{\text {eff }}=\left(4 \pi l Z^{2} N_{\mathrm{col}} / l_{g}\right)^{-1 / 2}$, as 


$$
z^{*}=\lambda_{\text {salt }}\left(-a_{1}+a_{2} \frac{l_{g}}{\lambda_{\text {col }}^{\text {eff }}}\right)
$$

with $a_{1}=\sqrt{4 \pi} c_{1}=0.5 \pm 0.2$. We do not consider here boundary effects: this equation is only valid if $z^{*}$ is smaller than $h$. The finite height $h$ of the container will have the effect to "push" the negative cloud downwards if the parameters are such that $z^{*}$ approaches the top of the container. The same holds for the bottom of the container if $z^{*}$ is too small.

Another quantity of interest is the size $\Delta z^{*}$ of the negative cloud, defined as the mid-height width of the negative peak in the charge density profile (see figure 1). Since we know that at $z^{*}$ altitude, $Z^{2} \rho_{\text {col }}\left(z^{*}\right)$ is of the same order of magnitude as $\rho_{+}\left(z^{*}\right)+\rho_{-}\left(z^{*}\right)=2 C_{\text {salt }}$, the screening length at that altitude is proportional to $\lambda_{\text {salt }}$. From basic electrostatics we know that the system will only tolerate charges over a length of order of the screening length, we deduce that $\Delta z^{*}$ is proportional to $\lambda_{\text {salt }}$. In fact the numerical results suggest also a linear dependency of $\Delta z^{*}$ in $l_{g}$ :

$$
\Delta z^{*}=b_{1} l_{g}+b_{2} \lambda_{\text {salt }}
$$

with $b_{1}=5.0 \pm 0.5, b_{2}=0.7 \pm 0.2$, and the same restrictions concerning boundary effects as for the equation for $z^{*}$.

\section{B. The apparent mass}

As we mentioned before, at high altitudes (larger than $z^{*}$ ) the electric potential is almost constant and the electric field vanishes. From this it is clear that the colloidal density will decay as $\exp \left(-z / l_{g}\right)$ and there is no apparent reduced mass. Nevertheless let us notice that in the regime where the electric potential is almost constant in our calculations the corresponding colloidal volume fraction is smaller than $10^{-9}$. Such volume fractions cannot be measured experimentally. In practice the optical methods used in [1] allow to measure only volume fractions larger than $10^{-5}$. A possible explanation to the apparent mass observed in the experiments is that for volume fractions higher than $10^{-5}$ the asymptotic regime where the electric field vanish have not been reached yet: there is a residual electric field responsible of the observed reduced mass.

In order to test this hypothesis we made a log plot of several colloidal volume fraction profiles restricting the plot to volume fractions higher than $10^{-5}$ (Figure2). We computed the slope of the wing of the colloidal density to find an effective gravitational length $l_{g}^{*}$ which is higher than the actual gravitational length $l_{g}$ as observed in the experiments. Futhermore when we plot the colloidal volume fraction profile and the corresponding electric field profile together (Figure 3) we notice that for volume fractions higher than $10^{-5}$ the electric field is not zero.

The different plots in Figure 2 where obtained using different salt concentrations, so the sediment height (which is proportional to $z^{*}$ ) varies. In this case we found that the apparent mass is a decreasing function of the height of the sediment, in agreement with the experiments. However the sediment height can be changed by changing other parameters like the mean colloidal density or their valency $Z$. Computing the apparent gravitational length $l_{g}^{*}$ as defined before for other series of samples, we found that the apparent gravitational length $l_{g}^{*}$ does not depend on $Z$ or the mean colloidal density. In our model the ratio $l_{g}^{*} / l_{g}$ is only a function of the salt density. Figure (4) shows the ratio $l_{g}^{*} / l_{g}$ as a function of salt screening length $\lambda_{\text {salt }}$. 


\section{COMPARISON WITH PREVIOUS APPROACHES}

As mentionned in the introduction the model presented above has motivated several studies both numerically [2] and analytically [4, 5 . The purpose of this section is to compare our numerical results with the most achieved version of the theory presented in reference [5]. This theoretical approach is based on a constrained minimization of the free energy functional (2.2) assuming an exponential ansatz for the density profiles:

$$
\begin{aligned}
\rho_{\mathrm{col}}(z) & =\frac{N_{\mathrm{col}} a}{l_{g}} \exp \left(-a z / l_{g}\right) \\
\rho_{+}(z) & =C_{\text {salt }} \\
\rho_{-}(z) & =C_{\text {salt }}+\frac{Z N_{\mathrm{col}} b}{l_{g}} \exp \left(-b z / l_{g}\right)
\end{aligned}
$$

With this parametrization $a=M^{*} / M$ is the ratio of the reduced mass $M^{*}$ by the buoyant mass $M$ of a colloidal particle, $C_{\text {salt }}$ denotes the fixed salt concentration, and $N_{\text {col }}$ is the fixed overall colloidal density per unit area, i.e. $\int_{0}^{+\infty} d z \rho_{\text {col }}(z) \equiv$ $N_{\text {col }}$. The system considered in [5] is semi-infinite, $z=0$ corresponds to the bottom of the sample and $h=+\infty . a$ and $b$ are the two variational dimensionless parameters of the theory, and the equilibrium density profiles $\rho_{\text {col }}(z)$ and $\rho_{-}(z)$ correspond to the values of $a$ and $b$ that minimize the free energy functional (2.2). Following reference [5], the minimization conditions express:

$$
\begin{gathered}
b(a)=a\left(\frac{2}{\sqrt{1+(1-a) / \gamma}}-1\right) \\
Z b(a)-\kappa I\left(\frac{Z b(a)}{\kappa}\right)-\gamma+\frac{4 \gamma b^{2}(a)}{(a+b(a))^{2}}=0
\end{gathered}
$$

where $\gamma=\pi Z^{2} l N_{\text {col }} l_{g}$ is the coupling parameter ( $l$ is the Bjerrum length introduced previously), and $\kappa=C_{\text {salt }} l_{g} / N_{\text {col }}$ is the relative amount of added salt. Function $I$ is defined by $I(x)=\int_{0}^{x} d y(\ln (1+y)) / y$.

Although equations (4.2) require a numerical treatment, it is possible to extract asymptotic expressions when the coupling parameter $\gamma$ is vanishingly small (strong gravitational coupling regime) or large compared to unity (strong Coulomb coupling regime). Such an analysis is presented in reference [5], and we only reproduce here the main features. When gravitational coupling is strong, $\gamma \ll 1$, the reduced mass is given by $a \simeq 1-3 \gamma$ (forall values of the salinity $\kappa$ ) and therefore no mass reduction is observed in this regime (in agreement with the numerical results presented in reference [2]). On the contrary, in strong Coulomb coupling regimes, $\gamma \gg 1$, quite a large mass reduction is predicted, even in low salinity regimes $\kappa \ll 1$ (in such a situation the mass reduction is given by $a \simeq\left[1+\frac{\kappa}{2} \ln ^{2}\left(\kappa\left(1+\frac{1}{Z}\right)\right)\right] /(Z+$ 1) $+O(1 / \gamma)$ ). Our numerical results based on a free minimization of the functional (2.2) show that it is indeed not the case, even though we observe nice exponential asymptotic behaviours at high altitudes. To emphasize this point we present in table 1 data obtained mostly in the strong Coulomb coupling regime. Excepted for the first value $\gamma=7.210^{-3}$, which corresponds to the opposite strong gravitational coupling regime, the agreement between the theory presented in reference [5] and the present free minimization is very poor. The effective mass predicted by the free minimization procedure corresponds to the actual mass within less than $0.1 \%$ in most situations, whereas the theory predicts effective masses that are only $2 \%$ of the actual mass! 
To our opinion, the failure of the parametrization presented in reference [5] is not due to the exponential ansatz itself, but to the constraint of global charge neutrality applied to the asymptotic regime. The theory presented in reference [5] assumes that the profiles are exponential from the bottom of the sample to the top, as a result the free energy functional (2.2) has to be minimized with the constraint of global charge neutrality. However, the actual situation is quite different. If we refer to the experimental work done by Piazza et al. [1], the exponential regime is reached only above a macroscopic layer of strongly interacting colloidal particles. Data presented in the previous section (see e.g. figure 11) resulting from a free minimization of the functional also exhibit a dense macroscopic layer of colloidal particles in the bottom of the cell, and these profiles cannot be simply represented by a single exponential. This feature can be incorporated to the model suggested by Löwen, by splitting the cell into two parts. The upper part of the cell (above a given altitude " $z_{o}$ ") corresponds to the asymptotic region where the profiles can be accurately represented by an exponential, whereas below $z_{o}$ the profiles are more complicated. As we can see, $z_{o}$ is defined by the condition that the profiles are exponential above it. There is then no upper bound on the value of $z_{o}$ and the asymptotic profiles should not depend on its precise value. As a result $z_{o}$ can be chosen arbitrarily large. As a consequence, the part of the fluid located below $z_{o}$ can be considered as a reservoir fixing the chemical potential of the ionic species $\mu_{\mathrm{col}}$ and $\mu_{-}\left(\mu_{+}\right.$is irrelevant since the local density $\rho_{+}(z)$ is held fixed, and is thus not a variational parameter). Although the full system must be charge neutral, the asymptotic part above $z_{o}$ has no reason to be neutral. We are then lead to minimize the free energy of the upper part of the cell in the grand-canonical ensemble. Assuming that parametrization (4.1) is valid above $z_{o}$ the minimization equation associated to the colloidal particles reads:

$$
\frac{\partial \mathcal{F}\left[\rho_{\mathrm{col}}, \rho_{+}, \rho_{-}\right]}{\partial a}=\mu_{\mathrm{col}} \frac{\partial N_{\mathrm{col}}}{\partial a}
$$

where $\mathcal{F}\left[\rho_{\text {col }}, \rho_{+}, \rho_{-}\right]$is now the free energy functional above $z_{o}$ :

$$
\begin{aligned}
\mathcal{F}\left[\rho_{\mathrm{col}}, \rho_{+}, \rho_{-}\right]= & \sum_{i \in\{\mathrm{col},+,-\}} \int_{z_{o}}^{+\infty} k_{\mathrm{B}} T \rho_{i}(z)\left[\ln \left(\lambda_{i}^{3} \rho_{i}(z)\right)-1\right] d z \\
& +\int_{z_{o}}^{+\infty} M g z \rho_{\mathrm{col}}(z) d z+\frac{1}{2} \int_{z_{o}}^{+\infty} e \rho(z) \Phi(z)
\end{aligned}
$$

and $N_{\text {col }}=\int_{z_{o}}^{+\infty} \rho_{\text {col }}(z) d z$ is the number of colloidal particles above $z_{o}$ per unit area. After some algebra, this minimization equation can be written on the form:

$$
\begin{aligned}
& (a-1)\left\{1+z_{o}^{*}+z_{o}^{* 2}\right\}+a\left\{\frac{\mu_{\mathrm{col}}}{k_{B} T}-\ln \left(\lambda_{\mathrm{col}}^{3} \frac{N_{\mathrm{col}} a}{l_{g}}\right)\right\} z_{o}^{*} \\
& +\gamma\left[e^{-z_{o}^{*}}\left(1+2 z_{o}^{*}\right)+4 e^{-z_{o}^{*} b / a}\left(\frac{a^{2}}{(a+b)^{2}}-\frac{1}{2}-\frac{z_{o}^{*}\left(a^{2}+b^{2}\right)}{b(a+b)}\right)\right]=0
\end{aligned}
$$

where $z_{o}^{*} \equiv a z_{o} / l_{g}$. We can easily check that when $z_{o}^{*}=0$ we recover the first equation of condition (4.2). As $z_{o}^{*}$ can be chosen arbitrarily large in our model, we easily see that this equation implies $a=1$ (no mass reduction) and:

$$
\frac{\mu_{\mathrm{col}}}{k_{B} T}=\ln \left(\lambda_{\mathrm{col}} \frac{N_{\mathrm{col}}}{l_{g}}\right)
$$

This new version of the theory is consistent with our numerical results predicting no mass reduction. 


\section{CONCLUSION}

A free minimization of the Poisson-Boltzmann theory used in references [2,5] have been performed in this article which lead us to conclude that this simple mean field theory does not predict any mass reduction contrarily to previous approximate minimization of the same functional. These new results are fully consistent with the analytical results obtained in a two-dimensional case by Téllez [3]. In particular, we observe the same condenser effect between the bottom of the container and the top of the dense region, resulting from a competition between electroneutrality and entropy of the microions. Data plotted in figure 2 give a possible explanation for the experimental results obtained by Piazza et al. [1]. Although in the asymptotic regime we observe no mass reduction, this regime is attained for very low values of the colloidal packing fractions, below the experimental resolution $\left(10^{-5}\right)$ in some situations. As a result, the residual electrostatic field can affect the profiles resulting in an apparent effective mass. 

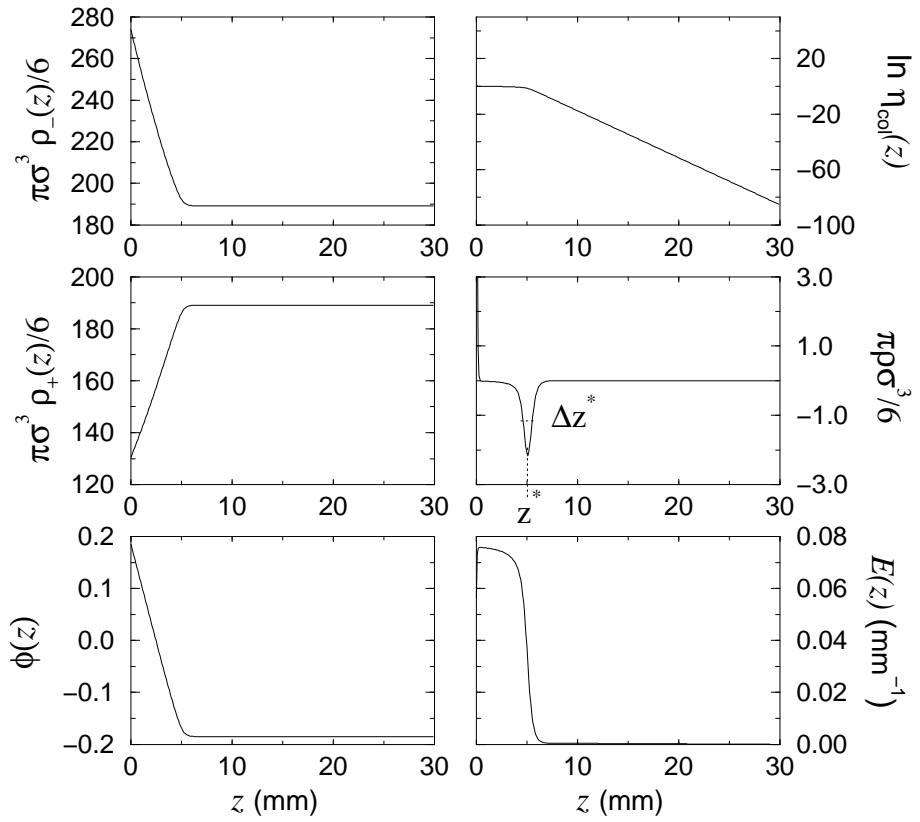

FIG. 1. From left to right, up to down, starting at the upper left corner, profiles of: counterions density, colloidal volume fraction in natural logarithm scale, coions density, charge density, dimensionless electric potential $\phi$, electric field $E=-d \phi / d z$. The parameters used are: $l=7 \cdot 10^{-8} \AA, l_{g}=0.128 \mathrm{~mm}, h=30 \mathrm{~mm}, Z=100, C_{\text {salt }}=0.1 \mathrm{mMol} / \mathrm{l}$, $\bar{\eta}_{\mathrm{col}}=0.12$ and $\sigma=180 \mathrm{~nm}$. 




FIG. 2. Colloidal density profile in decimal logarithm scale for different salt concentrations and restricted to volume fractions higher than $10^{-5}$. Common parameters to all curves are: $l_{g}=0.128 \mathrm{~mm}, Z=100, \sigma=180 \mathrm{~nm}, \bar{\eta}_{\text {col }}=0.12$ and $h=30 \mathrm{~mm}$. The salt concentration in $\mathrm{mMol} / \mathrm{l}$ from left to right is $0.4,0.3,0.2,0.1,0.05,0.04,0.03,0.02,0.01$, 0.005. The apparent gravitational length $l_{g}^{*}$ obtained from the slope of the low density wing is, from left to right in $\mathrm{mm}: 0.131 \pm 0.003,0.131 \pm 0.003,0.134 \pm 0.003,0.140 \pm 0.004$, $0.151 \pm 0.012,0.155 \pm 0.12,0.162 \pm 0.013,0.172 \pm 0.014,0.187 \pm 0.009,0.217 \pm 0.011$. 


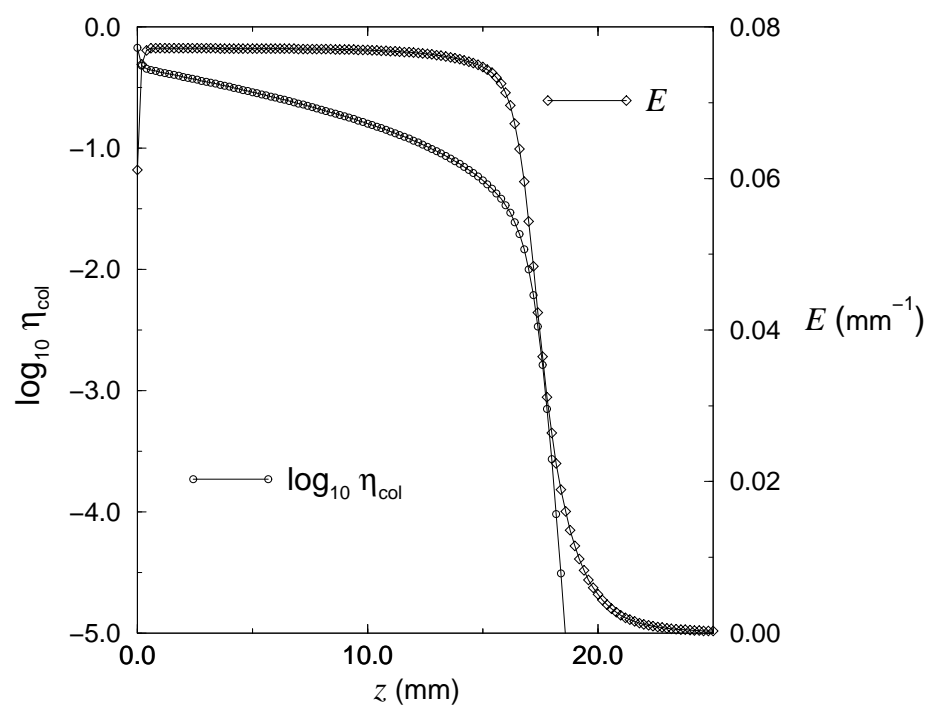

FIG. 3. Colloidal volume fraction decimal logarithmic profile and the corresponding electric field profile in the case $C_{\text {salt }}=0.005 \mathrm{mMol} / \mathrm{l}$, the other parameters being those of figure 2. Notice that in the low density wing used to compute the apparent gravitational length $l_{g}^{*}$ the electric field is not zero.

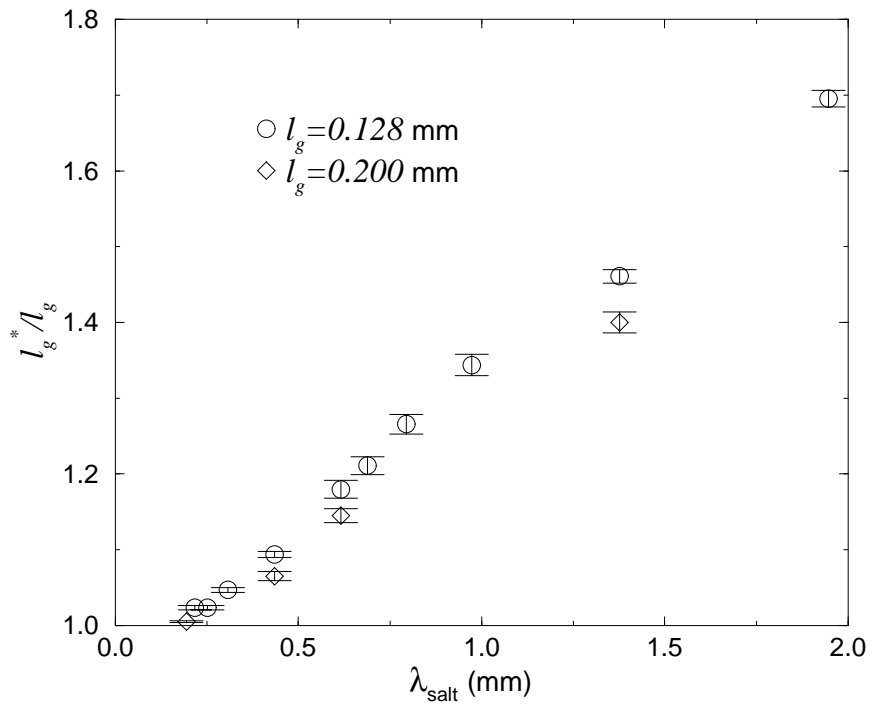

FIG. 4. The ratio of apparent gravitational length by the actual gravitational length $l_{g}^{*} / l_{g}$ versus the salt screening length $\lambda_{\text {salt }}$, for two different values of the gravitational length. 


\begin{tabular}{|c|c|c|c|c|c|}
\hline \hline$\gamma$ & $\kappa$ & $C_{\text {salt }}$ & $Z$ & $M^{*} / M:$ Theory & $M^{*} / M$ : free minimization \\
\hline $7.210^{-3}$ & $610^{4}$ & $3.2710^{-4} \mathrm{Mol} / \mathrm{l}$ & 100 & 0.9915 & $0.9994 \pm 0.0001$ \\
\hline 33.2 & 0.33 & $510^{-6} \mathrm{Mol} / \mathrm{l}$ & 100 & 0.0204 & $0.986 \pm 0.015$ \\
\hline 33.2 & 6.5 & $10^{-4} \mathrm{Mol} / \mathrm{l}$ & 100 & 0.0590 & $0.999 \pm 0.001$ \\
\hline 33.2 & 325 & $510^{-3} \mathrm{Mol} / \mathrm{l}$ & 100 & 0.3103 & $1.00001 \pm 0.00001$ \\
\hline 133 & 6.5 & $10^{-4} \mathrm{Mol} / \mathrm{l}$ & 200 & 0.0296 & $0.9998 \pm 0.0003$ \\
\hline \hline
\end{tabular}

TABLE I. Comparison between the reduced mass predicted by Löwen's theory and our numerical results from the free minimization.

[1] R. Piazza, T. Bellini, and V. Degiorgio, Phys. Rev. Lett. 71, 4267 (1993).

[2] T. Biben and J. P. Hansen, J. Phys. C: Condens. Matter 6, A345 (1994).

[3] G. Téllez, J. Chem. Phys. 106, 8572 (1997). and G. Téllez, J. Phys. A: Math. Gen. 31, 5277 (1998).

[4] J. P. Simonin, J. Phys. Chem. 99, 1577 (1995).

[5] H. Löwen, J. Phys.: Condens. Matter 10, L479 (1998).

[6] N. F. Carnahan and K. E. Starling, J. Chem. Phys. 51, 635 (1969).

[7] J. P. Badiali, M. L. Rosinberg, D. Levesque and J. J. Weis, J. Phys. C: Solid State Phys. 16, 2183 (1983). 